\title{
Plasticity of the tannosome ontogenesis in the Tracheophyta
}

\author{
Jean-Marc Brillouet \\ UMR SPO INRA/SupAgro/UM I, Montpellier, France
}

\section{Email address:}

brilloue@supagro.inra.fr

\section{To cite this article:}

Jean-Marc Brillouet. Plasticity of the Tannosome Ontogenesis in the Tracheophyta. Journal of Plant Sciences. Vol. 2, No. 6, 2014, pp. 317-323. doi: 10.11648/j.jps.20140206.19

\begin{abstract}
The tannosome, an organelle producing condensed tannins in the chlorophyllous organs of the Tracheohyta, is formed from the unstacked granal thylakoids by pearling into tiny spheres [1]. Other variants of intraplastidial ontogenesis of the tannosomes were investigated in the Tracheophyta by transmission electron microscopy. The "pearl necklace" model consists in the formation of vaguely ellipsoidal loculi by inflation of the intrathylakoidal lumen in which emerge, bound to the inner face of the lumen, "pear necklace"-shaped suites of osmiophilic spheres $(30 \mathrm{~nm})$; once filled with tannins, these loculi can be described as giant tannosomes bound by a single thylakoidal membrane. In the "snail" model, the granal thylakoids whirl upon themselves while pearling tannosomes which remain, in the stroma, aggregated in vaguely spherical osmiophilic structures. While in the former model [1], numerous isolated tannosomes are packed in a shuttle, the aggregated tannosomes ("pearl necklace" and "snail" models) are ejected from the chloroplast by budding into shuttles.
\end{abstract}

Keywords: Tannosome, Tracheophyta, Proanthocyanidins, Condensed Tannins, Polymerization

\section{Introduction}

Condensed tannins, also called proanthocyanidins, are present in most Tracheophyta. They are polymers of flavan-3-ols linked through C4-C6 and C4-C8 linkages. They are known for long to be stored in the vacuole. Early works have suggested that flavonoids, the supposed polymer building units, are synthesized from phenylpropanoids by a multi- enzymatic complex loosely bound to the cytosolic face of the endoplasmic reticulum [1-4]. However, the site of their poly- merization into condensed tannins was not known [5] until we recently published a complete description of the tannosome ontogenesis in the Tracheophyta [1]: briefly, plastidial thylakoids unstack, swell, then generate in the stroma tiny spheres, the tannosomes, in which polymerization occurs. These new organelles are then packed into shuttles which convey them to the vacuole. Shuttles then aggregate into chlorotannic accretions [6].

In view of our experience and although the thylakoidal origin of tannin polymers is not questioned, it appears that the tannosome ontogenesis is versatile and that several modes of formation coexist in the chloroplast with the first model [1]. A detailed morphological and ultrastructural study was carried out to examine the plasticity of the tannosome formation in diverse Tracheophyta.

\section{Materials and Methods}

\subsection{Plant Materials}

Leaflets from Cycas revoluta Thunb. (Cycadophyta), and persimmon (Dyospiros kaki L., Magnoliophyta, Eudicots), petioles from Chamaerops humilis L. (Magnoliophyta, Monocots), and fruits from grapevine (Vitis vinifera L., Magnoliophyta, Eudicots) were collected in the Montpellier City botanical garden.

\subsection{Transmission Electron Microscopy}

Specimens were dipped in $50 \mathrm{mM}$ sodium cacodylate buffer ( $\mathrm{pH} 7.0)$ containing 6\% glutaraldehyde (w/v) [7] and 1\% caffeine $(\mathrm{w} / \mathrm{v})$ for $6 \mathrm{~h}$, then treated with $1 \%$ osmium tetroxide $(\mathrm{w} / \mathrm{v})$ in water for $1 \mathrm{~h}$. After dehydration, they were embedded in Epon EmBed 812. Sections were stained with 0.2\% Oolong tea [8]. Ultrathin sections $(60 \mathrm{~nm})$ were visualised by an $\mathrm{H}-7100$ Hitachi transmission electron microscope with $75 \mathrm{kV}$ accelerating voltage. 


\section{Results}

\subsection{The "Pearl Necklace" Model}

Examination of thousands of sections from young organs of
Tracheophyta revealed, aside photosynthetic chloroplasts, plastids bearing in their inner volume numerous vaguely ovoid loculi formed, when enough space was available, between thylakoidal membranes on the edge of the grana (Plate 1A).

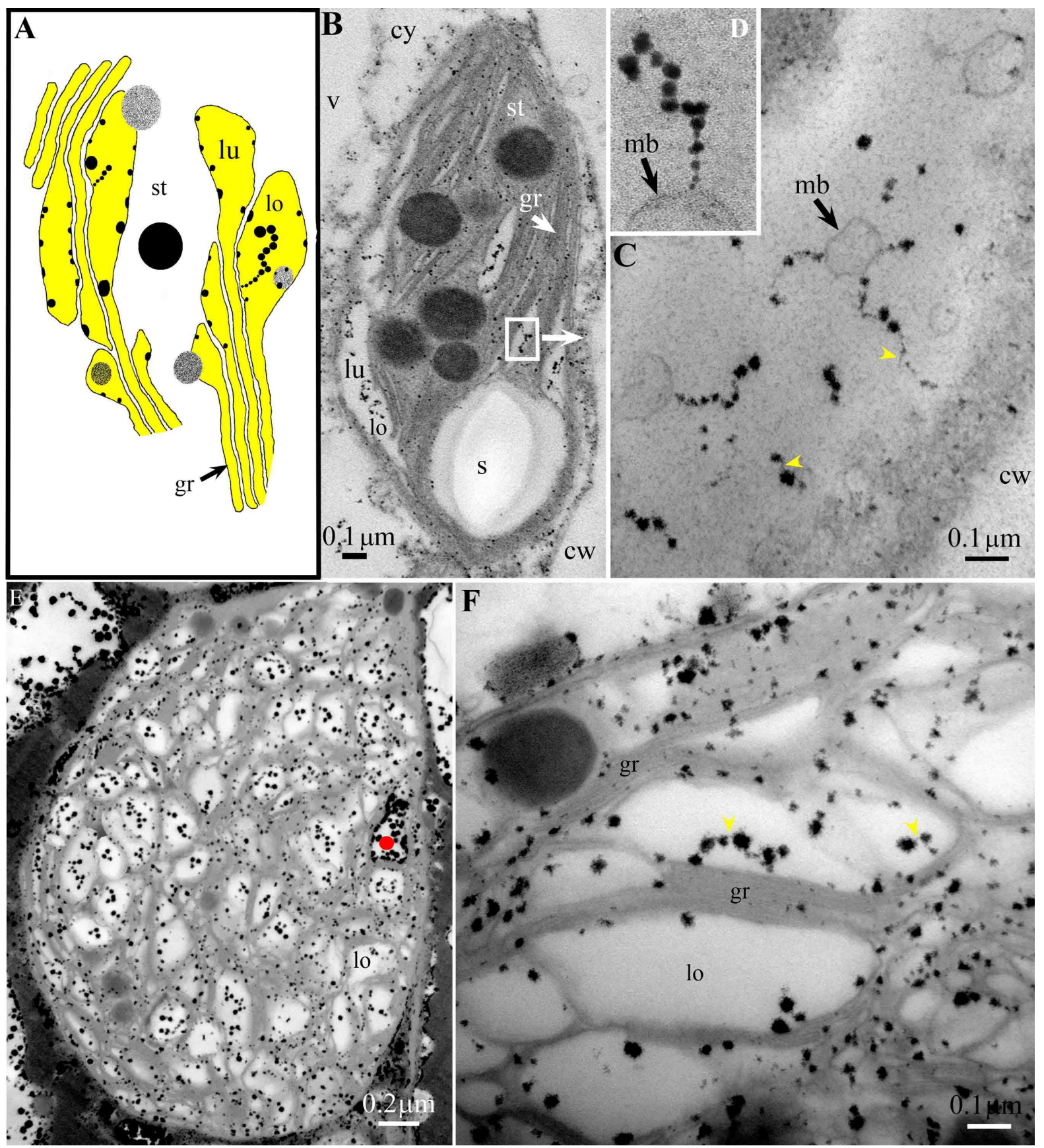

Plate 1. Evolving thylakoids in the "pearl necklace" mode. (E) A hardly recognizable chloroplast entirely filled with loculi; note a more advanced locule (red dot). Thin filament (yellow arrowheads). (A) Model; (B-D) Vitis; (E,F) Chamaerops. cw, cell wall; gr, granum; lo, locule; lu, lumen; mb, membrane; s, starch; st, stroma.

Bound to the inner face of membranes surrounding these loculi were seen small and finely granular black dots (diameter $<30 \mathrm{~nm}$ ) as well as osmiophilic large spheroids (Plate 1B-F). These spheroids were either contained in the thylakoidal lumen, or budding into the stroma, or even entirely bathing in the stroma. The black dots were seen in diverse situations: they were single, lining the inner face of the thylakoidal membrane of the locule, and also under the form of a chain carrying, at regular intervals, spheroids of which the diameter increases from the membrane outwards (maximum diameter observed 30nm) (Plate C,F); a very typical "pearl necklace"-shaped structure is illustrated (Plate 1D). Their density in loculi was variable, from one dot to tenths of them (Plate 1E), as were the shape and volume of loculi. 
Another feature of this model is that adjacent loculi can evolve to mostly circular multi-loculi chambers (Plate 2D, E). chloroplastsevolving in the "pearl necklace" model, with one spectacular locule budding off a Cycas chloroplast (Plate2B).

In Plate 2, are presented diverse morphologies of
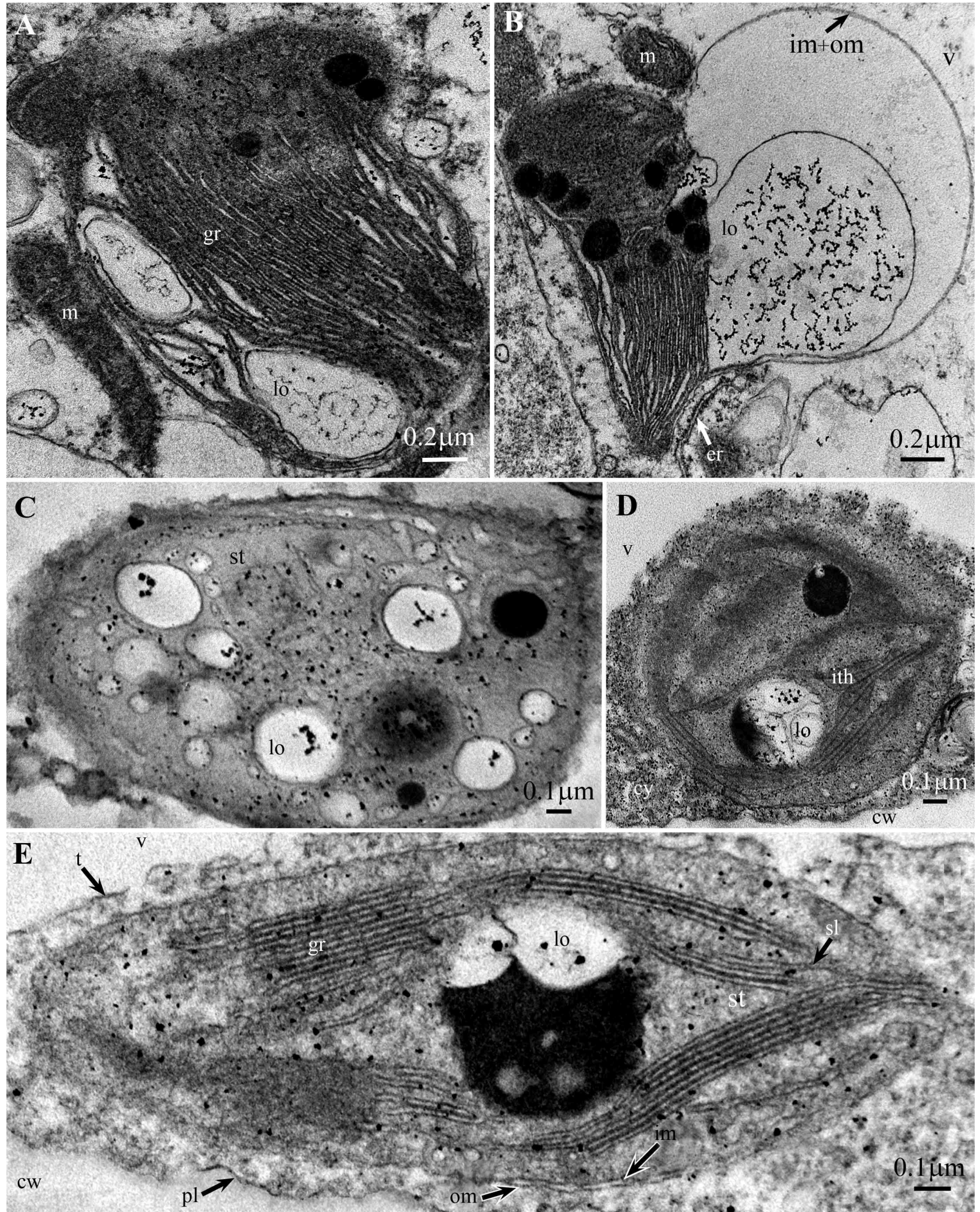

Plate 2. Chloroplasts evolving in the "pearl necklace" mode; note in $(D, E)$ the multi-loculi chambers. $(A, B)$ Cycas; $(C, E)$ Vitis; $(D)$ Dyospiros. cw, cell wall; cy, cytosol; er, endoplasmic reticulum; gr, granum; im, inner membrane; ith, inflated thylakoids; lo, loculus; om, outer membrane; m, mitochondrion; pl, plasmalemma; sl, stromal lamella; st, stroma; $t$, tonoplast. 


\subsection{The "Snail" Model}

Another situation was encountered (Plate 3): thylakoids, instead of pearling tannosomes from their end into the stroma [1], whirled on themselves generating tannosomes in a "snail"-shaped pattern (Plate 3A-D). These figures increased in diameter up to $0.8 \mu \mathrm{m}$, some of them showing incomplete filling at their periphery or in their body (Plate 4E,G). After completion of the filling, the thylakoidal net disintegrated into free tannosomes and fragments of thylakoids (Plate 4F-H).

\subsection{Fate of the Tannosomes}

Whatever their mode of formation, tannosomes aggregated in the stroma were expulsed out of the chloroplast in shuttles similar to those described in the former model [1] (Plate 4).

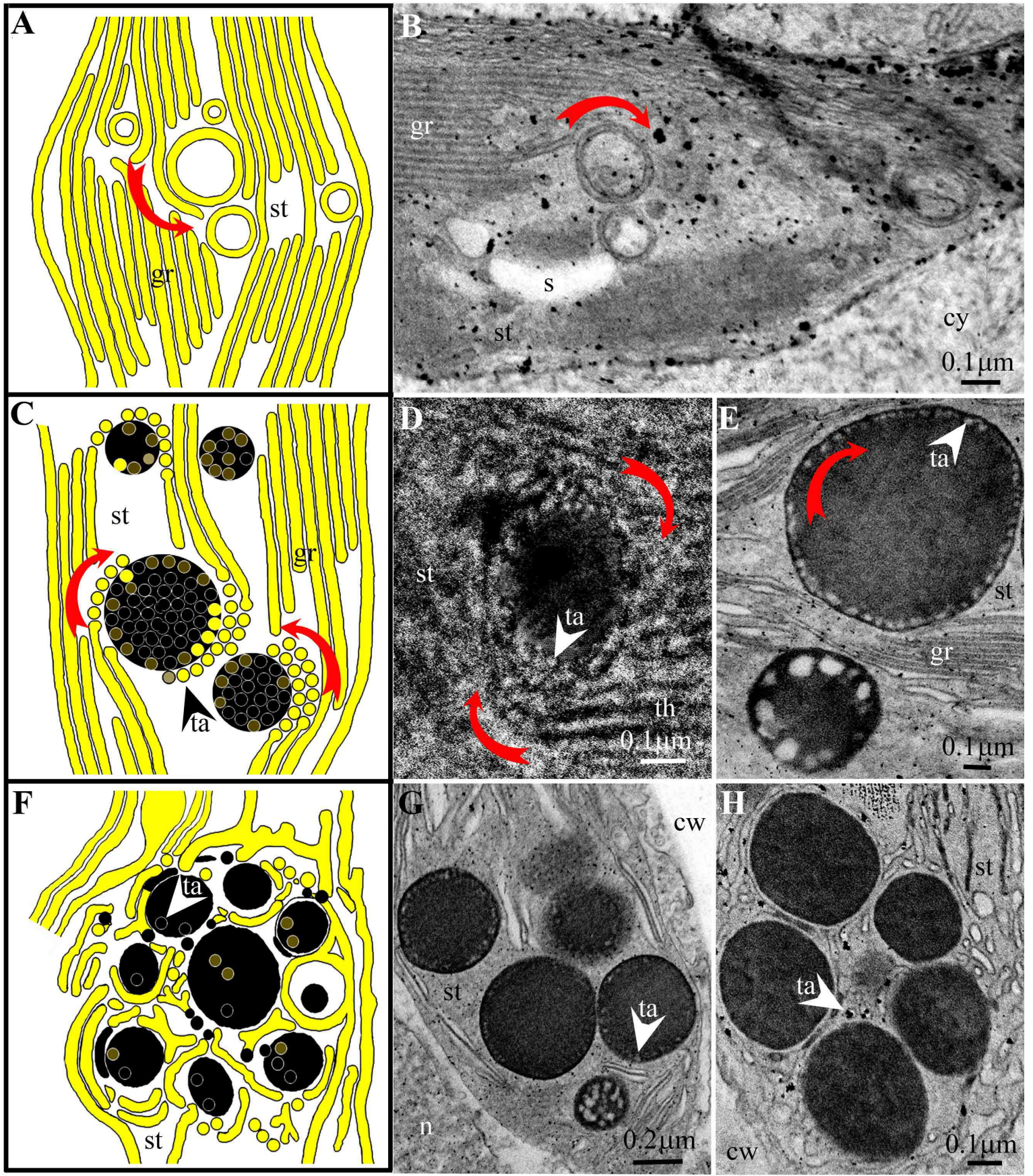

Plate 3. Evolving thylakoids in the "snail" mode. $(A, B)$ Beginning of whirling; $(C, E)$ generation of tannosome in an encircling movement; $(F, H)$ completion of filling and disintegration. (B,H) Vitis; (D) Dyospiros; (E,G) Chamaerops. cw, cell wall; gr, granum; s, starch; st, stroma; ta, tannosome; th, thylakoid. 

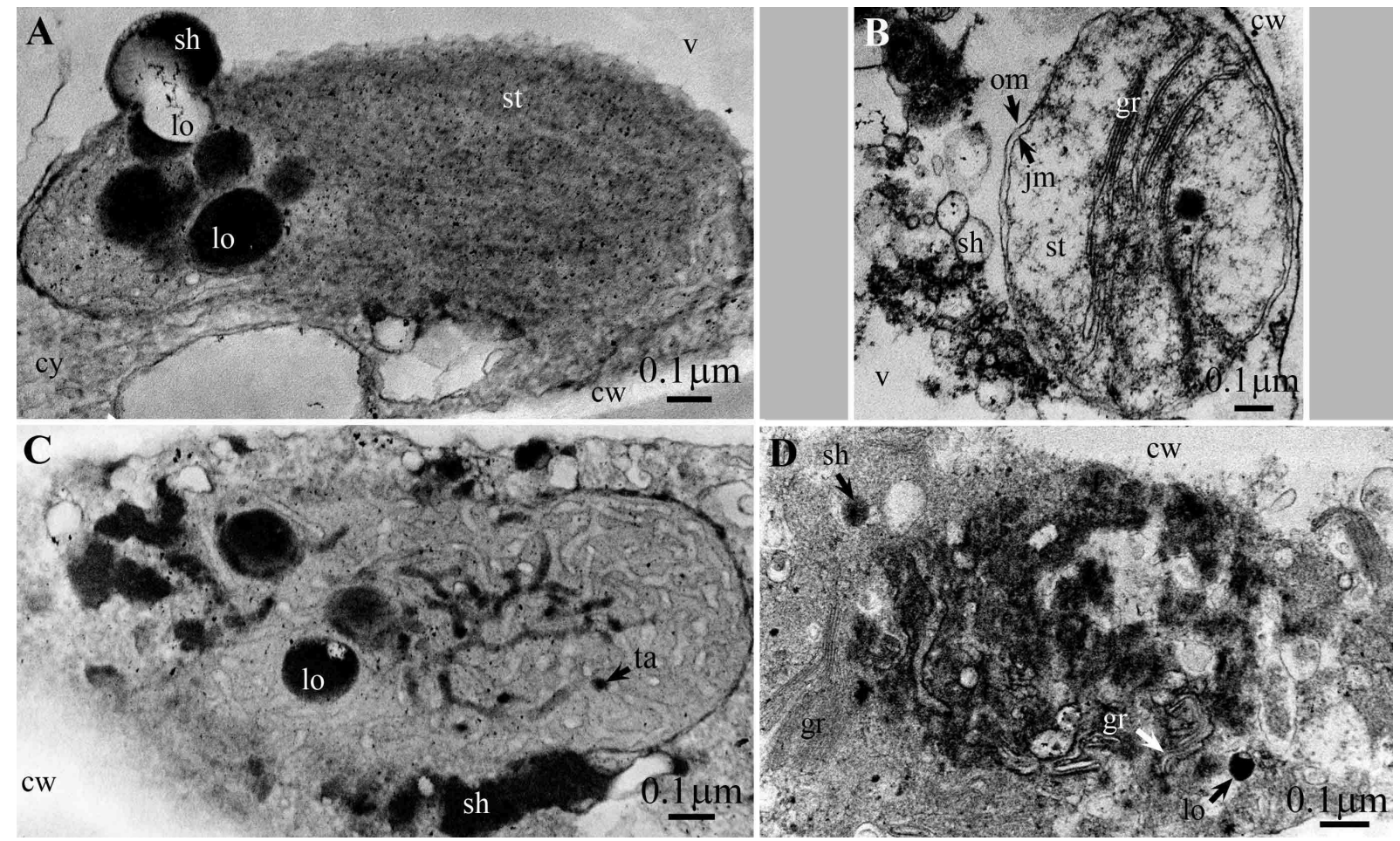

Plate 4. Chloroplasts forming loculi and emitting shuttles. Note disintegration in (D). $(A, C)$ Vitis; $(B, D)$ Cycas. cw, cell wall; cy, cytosol; gr, granum; im, inner membrane; lo, locule; om, outer membrane; sh, shuttle; st, stroma; v, vacuole.

\section{Discussion}

Thus, it appears that ontogenesis of the tannosome can follow diverse routes:

\subsection{The Tannosome Model [1]}

When, in the granum, intrathylakoidal lumen volume is limited, unstacked granal lamellae swell at very regular intervals generating a sequence of spherical structures $(30 \mathrm{~nm}$ diameter), the tannosomes; these chains can be seen in both the redifferentiating chloroplast and shuttles ([1]; Figs. 4B,C and 5B,C). While still in the thylakoids or after their separation, individual tannosomes produce, from the inner face of their thylakoidal membrane, osmiophilic dots which, in the end, fill their whole inner volume ([1]; Figs. 4H,I); it must be noted that the original black dots in the thylakoids are not linked together but rather attached to their parent membrane through a small pedicel.

\subsection{The "Pearl Necklace" Model}

After thylakoids have generated inflated chambers (loculi) leaving enough space in their lumen, osmiophilic spheres engendered from the inner face of the lumen membrane and connected by a thin osmiophilic junction protrude into the lumen area with a characteristic "pearl necklace" appearance (Plate 1). The intervals between these spheres are the same as the distances between inflated zones of the thylakoids forming tannosomes ([1]; Fig. 5C); moreover, the maximum diameter reached by these spheres is similar to the tannosome diameter, i.e. $30 \mathrm{~nm}$. So, in that case, these chambers can be viewed as giant tannosomes bound by a single thylakoidal membrane; according to the former model, thousands of tannosomes are bound by such a membrane.

Of utmost importance although not understood, is the progressive increase of pearls diameter from the membrane outwards (Plate 1D); these "necklace" structures must not be mistaken with polysomes [9] since ribosomes show a constant (not variable) diameter of 15-17 nm. After thylakoids have generated inflated chambers (loculi) leaving enough space in their lumen, osmiophilic spheres engendered from the inner face of the lumen membrane and connected by a thin osmiophilic junction protrude into the lumen area with a characteristic "pearl necklace" appearance (Plate 1). The intervals between these spheres is the same as the distances between inflated zones of the thylakoids forming tannosomes ([1]; Fig. 5C); moreover, the maximum diameter reached by these spheres is similar to the tannosome diameter, i.e. $30 \mathrm{~nm}$. So, in that case, these chambers can be viewed as giant tannosomes bound by a single thylakoidal membrane; according to the former model, thousands of tannosomes are bound by such a membrane.

Concerning the generation of loculi and subsequent emergence of "necklace" structures, we propose the following assessments and hypotheses.

The first assessment that could be made is that the polymerization of tannins is concomitant or follows the formation of intrathylakoidal inflated loculi: this phenomenon could result from a movement of water from the stromal medium into the lumen to dilute a yet unknown osmoticum. The second one is that the polymerization of tannins is an anisotropic phenomenon. Indeed, the process seems to be 
precisely structured both in space and time:condensed tannins are produced first as a single spheroid seemingly "extruded" from the inner face of the thylakoidal membrane into the lumen; this spheroid increases its volume while still attached to the membrane through a pedicel (see Plate 1D and [1], Fig. 4I). Then, other spheroids are sequentially produced from the same site of anchoring pushing the first older sphere into the lumen and, thus, form a "pearl necklace" structure; spheroids are equidistant, linked together by a thin filament, and their volume increases from the "youngest" to the "oldest".

So it appears that the condensed tannins are polymerized under two modes: either in individual tannosomes (one spheroid) [1] or in "pearl necklace" structures (several spheroids in file); both situations can coexist within a single shuttle (see [1], Fig. 5B). Several alternatives can be envisaged:

-the polymerization would entirely depend on the thylakoidal membrane: the substrate(s), as yet unknown, would arise from either the stroma or the lumen, and would be processed in the membrane. This hypothesis is compatible with the neoformed osmoticum possibly related to the substrate(s). In that case, one must question the role of the thin filament linking the spheroids to form the pearl necklace. This filament is present in single tannosomes as a short pedicel [1]; but, when still in the inflated portions of the unstacked thylakoids, they are not linked together; this means that, for their development, spheroids need to be linked to the membrane, either directly (single tannosome) or through the necklace filament (the "pearl necklace" model). Then arises a question: since, the diameter of spheroids increases from their point of attachment, would condensed tannins be extruded from the membrane and travel along the filament before deposition in the appropriate spheroid? The role of proteins in the structuration of the necklace has not yet been evoked: would there be any suicide proteins playing a role in the building of the necklace? What could be the role of lipids? Finally, why $30 \mathrm{~nm}$ would be a limiting diameter (single tannosomes or "necklace"-linked spheroids) while there is enough space in loculi to accommodate for larger spheroids?

-the polymerization would not depend on the thylakoidal membrane: the substrate(s) would be captured from the lumen liquid phase, polymerization occuring within spheroids (not in the membrane). This hypothesis is compatible with the neoformed osmoticum possibly related to the susbstrate(s). Coherence with the necklace structure could be commented as follows: if the necklace moves around its site of anchoring, then the "oldest" one will moves in a cone of a larger volume where more substrate(s) will be available than for the "youngest" one.

-the polymerization would apparently depend on the thylakoidal membrane, but the attachment of the first spheroid to the inner face of the membrane would be an artifact; indeed, since condensed tannins are known to form insoluble complexes with proteins [10], as soon as they will be formed in the first spheroid, they will "stick" to the membrane giving the false impression of a functional role of this membrane. However, the necklace structure contradicts this hypothesis.

Exceptionally well-preserved chloroplasts from Metasequoia fossil leaves found in Eocene Canadian high Arctic lignite showed such loculi containing vaguely granular osmium-positive materials which occasionally exhibited worm-like shape ([11]; Fig. 8); authors stated that these osmiophilic matters were lipid droplets (i.e. plastoglobules). It is very unlikely that lipids contained inside these globuli (tocopherol, carotenoids) [12] would have resisted degradation by the microflora along fossilization while condensed tannins would. Indeed, imperfect "pear necklace"-shaped chains similar to those observed in the present study were observed in Metasequoia (Plate 5). Moreover these loculi were not perfectly circular, which, according to their constitution, is a characteristic of plastoglobules [12]; it thus appears that these numerous chambers are of similar nature to those of modern Tracheophyta, i.e. intrathylakoidal tannin repositories. In all cases, it appears that the geometrical characteristics of the tannosome sequences, i.e. the thylakoids swollen at regular intervals, is not governed by the membrane itself but by the necklace operating unit.

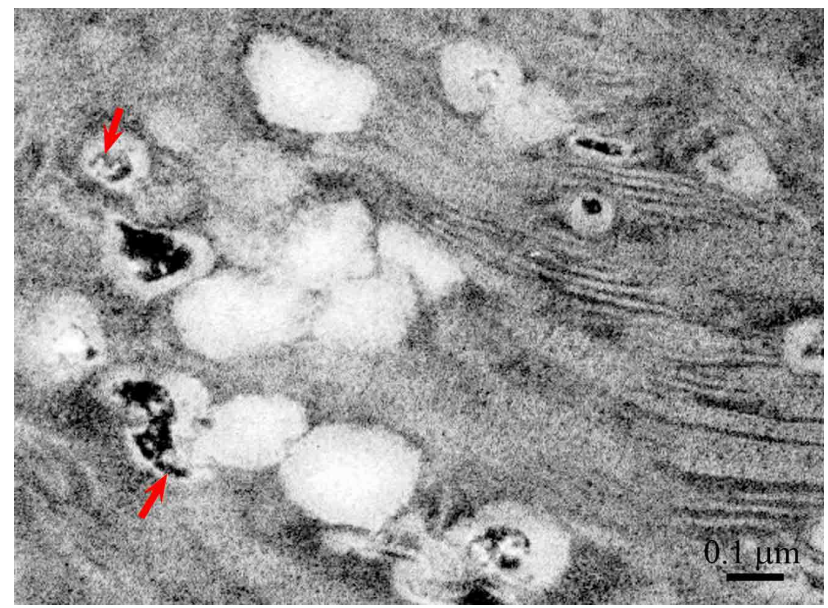

Plate 5. Leaf cross-sections and chloroplast ultrastructure in Eocene Metasequoia. From Ref. [11]. Osmiophilic "pearl necklace"-shaped inclusions in loculi (red arrows).

\subsection{The "Snail" Model}

This model differs from the first one [1] only by the fact that tannosomes are not free in the stroma but aggegrated in vaguely spherical objects.

\section{Conclusion}

Thus it appears that the modes of formation of tannosomes in the Tracheophyta are basically the same (Plate 6), but in the "necklace" model where giant instead of tiny tannosomes are formed. Their mode of expulsion from the tannin-forming chloroplasts through shuttlesremainsconstant. 


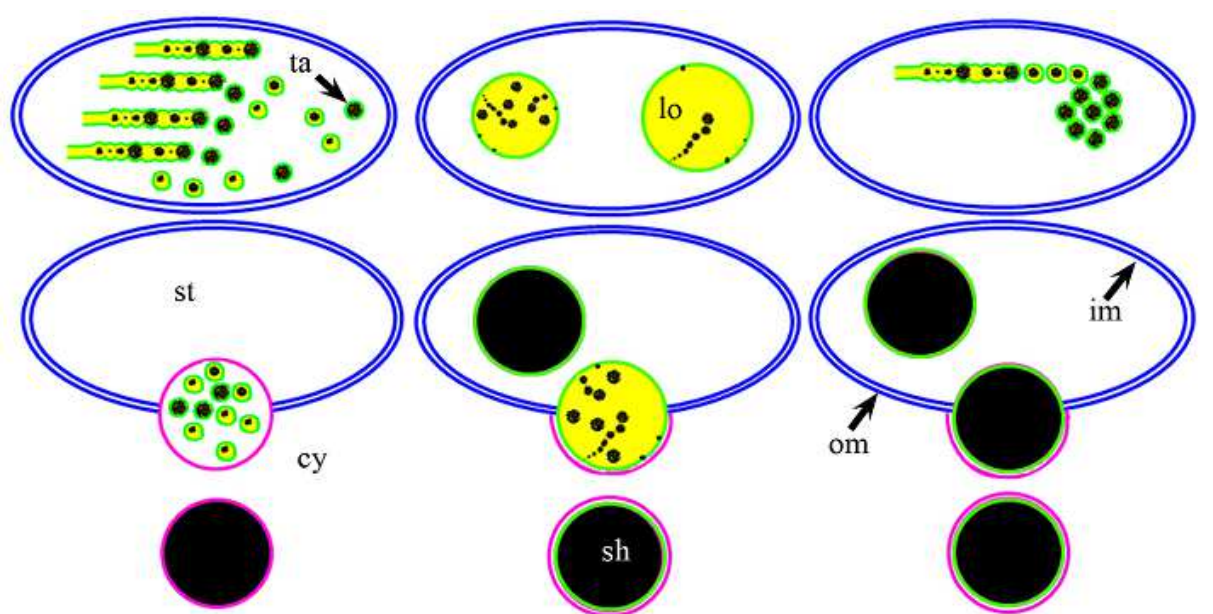

Plate 6. Models of tannosome generation in the Tracheophyta (not on scale). im, inner membrane; cy, cytosol; om, outer membrane; sh, shuttle; st, stroma; $\square$ intrathylakoidal lumen=locule; thylakoidal membrane; $\square$ shuttle membrane; plastidial outer and inner membranes, condensed tannins.

\section{Acknowledgments}

We thank Dr Chantal Cazevieille and Cécile Sanchez [Centre de Ressources en Imagerie Cellulaire (CRIC), Université Montpellier I, Montpellier, France], for their technical assis tance in transmission electron microscopy, and Dr Charles Romieu (INRA, AGAP, Montpellier, France) for helping to make significant improvements to this paper.

\section{References}

[1] J.-M. Brillouet, C. Romieu, B. Schoefs, K. Solymosi, V. Cheynier, H. Fulcrand, J.-L. Verdeil, and G. Conéjéro. 2013. The tannosome is an organelle forming condensed tannins in the chlorophyllous organs of Tracheophyta. Ann. Bot. 112:1003-1014 11003-1014.

[2] G. J. Wagner and G. Hrazdina. 1984. Endoplasmic reticulum as a site of phenylpropanoid and flavonoid metabolism in Hippeastrum. Plant Physiol. 74: 901-906.

[3] G. Hrazdina, A. M. Zobel, and H. C. Hoch. 1987. Biochemical, immunological and immunocytochemical evidence for the association of chalcone synthase with endoplasmic reticulum membranes. PNAS USA 84: 8966-8970.

[4] I. E. Burbulis, and B. Winkel-Shirley. 1999. Interactions among enzymes of the Arabidopsis flavonoid biosynthetic pathway. PNAS USA 96: 12929-12934.

[5] J. Zhao, Y. Pang, and R. A. Dixon. 2010. Update on biosynthesis of proanthocyanidins. The mysteries of proanthocyanidin transport and polymerization. Plant Physiol. 153: 437-443.

[6] J. M. Brillouet, C. Romieu, M. Lartaud, E. Jublanc, L. Torregrosa, and C. Cazevieille. 2014. Formation of vacuolar tannin deposits in the chlorophyllous organs of Tracheophyta: from shuttles to accretions. Protoplasma 251: 1387-1393.

[7] C. Sironval, R. Kirchman, R. Bronchart, and J. M. Michel. 1968. Sur le freinage de l'accumulation des chlorophylles dans les feuilles primordiales de Phaseolus vulgaris L. var. Commodore à la suite d'une irradiation ; photorestauration en lumière continue. Photosynthetica 2: 57-67.

[8] S. Sato, A. Adachi, Y. Sasaki, and M. Ghazizadeh. 2008. Oolong tea extract as a substitute for uranyl acetate in staining of ultrathin sections. J. Micros. 229: 17-20.

[9] H. Falk. 1969. Rough thylakoids: polysomes attached to chloro plast membranes. J. Cell. Biol. 42: 582-587.

[10] Hagerman AE, Butler HG. 1981. The specificity of proanthocyanidin-protein interactions. J. Biol. Chem. 256: 4444-4497.

[11] K. Schoenhut, D. R.Vann, and B. A. Lepage. 2004. Cytological and ultrastructural preservation in Eocene Metasequoia leaves from the Canadian high arctic. Amer. J. Bot. 91: 816-824.

[12] J. R. Austin II, E. Frost, P.-A. Vidi, F. Kessler, and L. A. Staehelin. 2006. Plastoglobules are lipoprotein subcompartments of the chloroplast that are permanently coupled to thylakoid membranes and contain biosynthetic enzymes. The Plant Cell 18: 1693-1703. 